\title{
Dermatite alérgica de contato a medicamentos de uso tópico: uma análise descritiva
}

\author{
Allergic contact dermatitis to topical drugs: a descriptive analysis
}

\author{
Rosana Lazzarini ${ }^{1}$ \\ Juliana Casagrande Tavoloni Braga ${ }^{3}$
}

\author{
Ida Duarte ${ }^{2}$ \\ Samia Leticia Ligabue ${ }^{4}$
}

\begin{abstract}
Resumo: FUNDAMENTOS: A dermatite de contato é uma dermatose comum e suas múltiplas causas dificultam seu tratamento.

OвjEтrvo: O objetivo do estudo foi avaliar a frequência da sensibilização aos medicamentos de uso tópico, em serviço universitário; estudar as características da população afetada e identificar os principais sensibilizantes relacionados aos medicamentos tópicos.

MÉTodos: Realizou-se um estudo retrospectivo dos pacientes com hipótese diagnóstica de dermatite alérgica de contato e que foram submetidos a testes epicutâneos. Avaliaram-se os que tiveram diagnóstico final de dermatite alérgica de contato a medicamentos tópicos.

Resultados: Em um grupo de 329 pacientes submetidos a testes de contato, 42 (13\%) tiveram testes de contato positivos e relevantes para medicamentos tópicos, pelo princípio ativo e/ou por outros componentes. Entre os testes positivos, 36 (85,7\%) corresponderam aos princípios ativos e 28 (66,7\%), a outros componentes das fórmulas, com alguns pacientes apresentando mais de um teste positivo. Entre os princípios ativos, a neomicina foi o mais frequente.

CONCLUSÕES: Entre os pacientes estudados, a sensibilização aos medicamentos tópicos ocorreu em $13 \%$ dos casos, com discreta preferência pelo sexo feminino e maior número de casos entre pacientes brancos. Houve elevada frequência de dermatite alérgica de contato pela neomicina, agente de uso comum como automedicação e também como prescrição médica.

Palavras-chave: Dermatite de contato; Dermatite de contato/etiologia; Eczema; Neomicina; Neomicina/efeitos adversos
\end{abstract}

\footnotetext{
Abstract: BACKGROUND: Allergic Contact Dermatitis is a common type of dermatitis, but its multiple etiologies hinder its management.

OвJестіvE: the objective of this study was to evaluate the frequency of sensitization to topical drugs in a university center, to study the main characteristics of the affected population, and to identify the allergens related with the dermatitis.

METHODS: a retrospective and descriptive study with patients submitted to patch tests. The study was conducted with subjects whose patch test to topical drugs was positive.

ReSULTS: 329 patients were submitted to patch tests and 42 (13\%) had positive and relevant patch tests to topical drugs. Among these patients, 36 (85.7\%) had positive patch tests to the principal component and 28 (66.7

$\%)$ to the other components. Some patients had more than one sensitization. Neomycin was the most important allergen.

Conclusions: Among the studied patients, sensitization to topical agents happened in $13 \%$ of the cases, with slight preference for females and Caucasians. There was elevated sensitization to neomycin, a popular agent among patients and medical doctors.

Keywords: Dermatitis, contact; Dermatitis, contact/etiology; Eczema; Neomicyn; Neomicyn/adverse effects
} 


\section{INTRODUÇÃO}

A dermatite de contato aos medicamentos tópicos pode ser iatrogênica ou induzida pelo próprio paciente em decorrência de automedicação. Eventualmente, tem caráter ocupacional, entre os profissionais da saúde ou da indústria farmacêutica que manipulam os medicamentos. ${ }^{1}$ A dermatite de contato por irritação primária (DCIP) por tópicos é desencadeada por agentes com propriedade de causar dano tecidual direto, ao passo que a dermatite alérgica de contato (DAC) resulta de sensibilização a uma substância que induz reação de hipersensibilidade mediada por células (tipo IV, de Gell e Coombs).

Clinicamente, o tipo mais frequente de reação a drogas de uso tópico é o eczema de contato, em todas as fases, sempre acompanhadas de prurido intenso. Em pacientes com dermatite de estase, não é rara a disseminação para outras áreas ou para todo o tegumento, levando à eritrodermia.

Os agentes etiológicos causadores da dermatite de contato, presentes na medicação de uso tópico, incluem tanto o princípio ativo quanto outros ingredientes (conservantes, acidulantes, emulsificantes), muitos dos quais são também encontrados nos cosméticos. ${ }^{1}$

A DCIP, muitas vezes, faz parte da ação terapêutica, em decorrência do uso da tretinoína, do peróxido de benzoíla ou 5-fluouracil. Outras reações, como a urticária de contato, a necrose cutânea (violeta de genciana, 5-fluouracil e polivinilpirrolidona iodo), a hiperpigmentação ou hipopigmentação (sais de mercúrio) e a dermatite de contato do tipo eritema multiforme (etilenodiamina, neomicina e sulfonamidas), podem ser observadas.

A DAC resultante de medicamentos, muitas vezes, apresenta dificuldade no diagnóstico etiológico devido ao uso de diferentes agentes tópicos pelo mesmo paciente. A incidência dessa dermatose varia de acordo com a área geográfica, pois depende de hábitos de prescrição, além de variações no decorrer dos anos. Alérgenos comuns há 20 ou 30 anos, como as sulfonamidas, penicilina e anti-histamínicos de uso tópico, foram substituídos por outras drogas, como os anti-inflamatórios não esteroidais (AINE) e os corticosteróides, que se tornaram novos alérgenos.

Alguns medicamentos tópicos também podem induzir a dermatite de contato fotoalérgica, que se expressa da mesma forma? eczema agudo ou subagudo, principalmente, em áreas expostas.

Os objetivos deste trabalho foram: 1) avaliar a frequência de sensibilização aos medicamentos de uso tópico, em um serviço universitário; 2) estudar as características da população afetada quanto a sexo, cor, idade e doença que levou ao uso da medicação e 3) verificar os principais sensibilizantes relacionados aos medicamentos tópicos.

\section{CASUÍSTICA E MÉTODOS}

Selecionaram-se para o estudo os pacientes que realizaram testes de contato no período entre janeiro de 2004 e março de 2006, no Setor de Alergia da Clínica de Dermatologia da Santa Casa de São Paulo, com suspeita de dermatite alérgica de contato. Retrospectivamente, escolheram-se os que apresentaram testes de contato positivos e relevantes a medicamentos de uso tópico, tanto ao princípio ativo quanto a outros componentes das fórmulas.

Para os testes, utilizou-se a Bateria Padrão Brasileira composta por 30 substâncias (FDA Allergenic Rio de Janeiro, Brasil). As substâncias que não faziam parte dessa bateria foram manipuladas de acordo com dados da literatura. ${ }^{2}$ Em todos os casos, usaram-se contentores do tipo FINN Chambers (Oy, Finland) e realizaram-se as leituras em 48 e 96 horas, de acordo com os critérios do International Contact Dermatitis Research Group (ICDRG) de 1981, em que: (-) reação negativa; reação duvidosa; $(+)$ reação fraca, com leve eritema e algumas pápulas; $(++)$ reação de média intensidade, com eritema, pápulas e algumas vesículas; $(+++)$ reação intensa, com eritema, pápulas e vesículas confluentes.

Os dados foram coletados com base em um protocolo utilizado na Clínica de Dermatologia, onde constam informações como idade, sexo, tempo de início da dermatose e resultados dos testes de contato. Nos casos necessários, os prontuários também foram avaliados. Inseriram-se esses dados em uma planilha Excel $^{\oplus}$ $\left(\right.$ Microsoft $\left.^{\circledR}\right)$, a partir da qual se quantificaram e se realizaram análises descritivas dos resultados.

\section{RESULTADOS}

No período de janeiro de 2004 a março de 2006 , entre os 329 pacientes submetidos aos testes de contato realizados no Setor de Alergia da Clínica de Dermatologia da Santa Casa de São Paulo, 42 (13\%) foram positivos e relevantes para medicamentos tópicos, pelo princípio ativo e/ou por outros componentes, dos quais 24 eram mulheres (57\%) e 18 eram homens (43\%). A distribuição por grupos étnicos mostrou 25 brancos (60\%), nove pardos (21\%), sete negros (17\%) e um amarelo (2\%). A faixa etária predominante foi entre 40 e 59 anos (18\% e 43\%, respectivamente).

Em dois pacientes (5\%), a dermatose teve início há menos de dois meses e, em 40 (95\%), a dermatose apresentava um período longo de evolução, variando entre três e 60 meses.

Quanto à localização, houve comprometimento dos membros inferiores em 22 pacientes (31\%), do segmento cefálico (região cervical e face) em 20 (25\%), dos membros superiores em 18 (26\%) e do tronco em cinco (7\%). Em oito casos, a dermatose era disseminada (11\%). Houve mais de uma região comprometida em cada paciente (Tabela 1).

Entre os testes de contato positivos, $36(85,7 \%)$ corresponderam aos princípios ativos e 28 (66,7\%), aos 
Tabela 1: Localizações mais frequentes da dermatite alérgica de contato por medicamento tópico

\begin{tabular}{lll}
\hline Localização & Número & Percentual \\
\hline Tronco & 5 & 7 \\
Membros superiores & 18 & 26 \\
Segmento cefálico & 20 & 25 \\
Disseminada & 8 & 11 \\
Membros inferiores & 22 & 31 \\
\hline Total & $73^{*}$ & $\mathbf{1 0 0}$ \\
\hline
\end{tabular}

* Alguns pacientes tiveram mais de uma área comprometida.

outros componentes das fórmulas, sendo que alguns pacientes tiveram mais de um teste positivo.

No que diz respeito aos princípios ativos, a neomicina teve 19 testes positivos (27\%), a prometazina, oito (12\%), a nitrofurazona, cinco (8\%), a quinolina, dois (3\%), a sulfadiazina de prata e o cloranfenicol, um teste cada (2\%). Estes dois últimos não fazem parte da bateriapadrão.

Outros componentes positivos foram: etilenodiamina e quaternium 15, com sete testes positivos cada (11\%); parabenos, em cinco (8\%); colofônio, em três (5\%); lanolina, em dois (3\%); irgasan, perfume mix, formaldeído e bálsamo do Peru, com um teste cada (2\% cada) (Tabela 2).

\section{DISCUSSÃO}

Os medicamentos de uso tópico são utilizados, muitas vezes, de modo indiscriminado, pela crença popular de causarem poucos efeitos colaterais. Por outro lado, a própria prescrição médica pode induzir a dermatite de contato, tornando-a uma dermatose iatrogênica.

Os pacientes com dermatoses crônicas, como dermatite atópica ou psoríase, têm maior propensão a desenvolver esse tipo de dermatite, devido à exposição a múltiplos agentes tópicos em pele, com perda da integridade.

Os casos de dermatite alérgica de contato aos medicamentos tópicos representaram $13 \%$ do total de pacientes testados, no período descrito, à semelhança do que foi encontrado em relatos da Europa, em que a incidência dessa dermatite em pacientes submetidos a testes de contato variou entre $13,2 \%$, na Itália, e $40 \%$, na Suécia. ${ }^{1}$

A incidência relaciona-se com o tipo de paciente estudado. Os portadores de dermatite de estase ou anogenital são de alto risco para ocorrência de reações às drogas tópicas, devido a condições que favorecem a penetração da medicação, como a alteração da barreira cutânea e o aumento da temperatura local. Nesses doentes, a incidência é alta e pode variar entre $58 \%$ e $86 \%$, dependendo da série estudada.
Os casos foram mais comuns entre as mulheres e entre pacientes de cor branca. Esses dados são compatíveis com outros já descritos na literatura, em que há prevalência aumentada de dermatite de contato entre pacientes do sexo feminino e em brancos.

Quanto às faixas etárias acometidas, notou-se uma ocorrência maior da dermatite após os 40 anos de idade. Nesse período da vida, há aumento no número de casos de dermatite alérgica de contato de modo geral, devido a maior exposição aos alérgenos. Além disso, aumenta a frequência tanto da dermatite quanto da úlcera de estase e dos eczemas crônicos e, portanto, cresce a exposição aos agentes tópicos.

A dermatite de contato teve evolução aguda, menor do que dois meses, em apenas dois casos (5\%), enquanto que, em $95 \%$ dos casos, a dermatose estava presente há mais de três meses. Esse fato reflete a dificuldade no diagnóstico etiológico da dermatose e o aumento da probabilidade de sensibilização às drogas tópicas em pacientes portadores de dermatoses que evoluem com perda da função de barreira da pele.

A dermatite alérgica de contato estava presente nos membros inferiores em 22 casos (31\%), a maior parte relacionada com a presença de dermatite de estase, dados que estão de acordo com os da literatura. Nesses casos, o sensibilizante mais comum foi a neomicina, seguida da etilenodiamina e da nitrofurazona. Houve, ainda, sensibilização ao bálsamo do peru, ao quaternium 15 , ao cloranfenicol, ao colofônio, a fragrâncias e à sulfadiazina de prata. Os dados de literatura também mostram como alérgeno mais frequente, nessa localização, a neomicina. Entretanto, os corticosteróides têm um papel importante como alérgenos em outras regiões do mundo, fato não documentado em nosso meio. Essas diferenças podem estar relacionadas aos hábitos regionais3.

A prometazina, cujos testes de contato foram positivos em $12 \%$ dos casos, é um anti-histamínico do grupo das fenotiazinas utilizado pelas vias oral, intramuscular e tópica. A última apresentação é de uso difundido em nosso meio e em alguns países da Europa, sendo capaz de desencadear DAC, além de reações fotoalérgicas e fototóxicas. 
TABela 2: Testes de contato positivos e relevantes para as substâncias presentes nos medicamentos tópicos

\begin{tabular}{lll}
\hline Substâncias & Número & Percentual \\
\hline Neomicina & 19 & 27 \\
Prometazina & 8 & 12 \\
Etilenodiamina & 7 & 11 \\
Quaternium 15 & 7 & 11 \\
Nitrofurazona & 5 & 8 \\
Parabenos & 5 & 8 \\
Colofônio & 3 & 5 \\
Quinolina & 2 & 3 \\
Lanolina & 2 & 3 \\
Sulfadiazina de prata & 1 & 2 \\
Cloranfenicol & 1 & 2 \\
Irgasan & 1 & 2 \\
Perfume mix & 1 & 2 \\
Formaldeído & 1 & 2 \\
Bálsamo do peru & 1 & $\mathbf{1 0 0}$ \\
\hline Total & $\mathbf{6 4 *}$ & \\
\hline
\end{tabular}

*Alguns pacientes tiveram mais de um teste positivo.

A nitrofurazona foi responsável por 14\% dos casos de sensibilização observados e é utilizada como antisséptico na forma de pomada ou como lubrificante em curativos para úlceras e queimaduras. Essa droga é empregada em nosso meio como material de curativo em serviços de urgência. Trata-se de um forte sensibilizante, capaz de causar casos graves de dermatite de contato4.

A quinolina foi responsável por $3 \%$ dos testes positivos. Esse antimicrobiano é utilizado em medicamentos tópicos, associado a corticosteróides, outros antibacterianos e antifúngicos. Em 1989, Goh, em Cingapura, encontrou $2,4 \%$ de sensibilização à quinolina entre 514 pacientes com DAC a antibióticos tópicos5. Os dados aqui apresentados mostram maior frequência de sensibilização, provavelmente, pelo uso mais difundido da medicação em nosso meio e também pela metodologia diferente empregada nos dois estudos.

Entre os pacientes analisados, observou-se um caso de DAC pela sulfadiazina de prata em portador de úlcera de estase do membro inferior, que evoluiu com fotossensibilidade. A sulfadiazina de prata é um agente antibacteriano tópico usado nas úlceras dos membros inferiores e em queimaduras químicas; sua propriedade antibacteriana se deve ao efeito combinado da prata e da sulfadiazina6. Trata-se de um sensibilizante pouco comum em nosso meio.

Um dos casos observados apresentou sensibilização ao cloranfenicol, antibiótico utilizado topicamente em colírios e em alguns cremes para ferimentos, quando costuma estar associado à colagenase. $\mathrm{O}$ caso observado foi submetido ao teste com a medicação como é vendida e com o cloranfenicol puro a $5 \%$ em vaselina sólida, com positividade a ambos. Casos de sensibilidade a esse agente são pouco comuns na Europa devido ao pouco uso. Em nosso meio, a frequência de sensibilização a esse agente é desconhecida.

Outros agentes presentes nas formulações dos medicamentos tópicos também foram positivos no grupo estudado, como etilenodiamina (emulsificante), quaternium 15, parabenos e formaldeído (conservantes), perfume mix, bálsamo do peru e colofônio (fragrâncias) e lanolina (veículo). Esses agentes são causadores frequentes de DAC, pois estão presentes em diversos produtos e devem ser lembrados e considerados no momento da interpretação dos resultados dos testes de contato. $^{8-11}$

Um fato interessante a ser destacado foi a presença prévia de eczemas de contato por outros agentes etiológicos, como esmalte de unhas, cimento e borracha, cujo diagnóstico não foi realizado inicialmente, o que levou ao uso de medicamentos tópicos de maneira inadvertida e causou a sensibilização secundária. Outras dermatoses, como psoríase, líquen simples crônico, eczema numular e dermatite atópica, também serviram de base para a DAC pelos medicamentos tópicos.

\section{CONCLUSÕES}

Observou-se, entre os pacientes estudados, que a dermatite alérgica de contato aos medicamentos tópicos ocorreu em 13\% dos casos, com discreta preferência pelo sexo feminino e maior número de casos entre pacientes brancos. A neomicina, agente de uso comum entre os pacientes e também como prescrição médica, foi o sensibilizante mais frequente. Esse fato deve alertar os médicos, especialistas ou não, no momento da prescrição, principalmente, para os portadores de dermatoses crônicas, os mais propensos a desenvolver dermatites alérgicas de contato a medicamentos de uso tópico. 


\section{REFERÊNCIAS}

1. Brandão FM, Goossens A, Tosti A. Topical drugs. In: Frosch PJ, Menné T, Lepoittevin JP. Contact Dermatitis. Speyer: Springer; 2006. p.623-52.

2. De Groot AC. Patch testing: concentration and vehicles for 3700 chemicals. Amsterdan: Elsevier Science; 1994.

3. Saap L, Fahim S, Arsenault E, Pratt M, Pierscianowski T, Falanga V. Contact sensitivity in patients with leg ulcerations. Arch Dermatol. 2004;140:124-6.

4. Prieto A, Baeza ML, Herrero T, Barranco R, De Castro FJ, Ruiz J. Contact dermatitis to Furacin. Contact Dermatitis. 2006;54:126.

5. Goh CL. Contact sensitivity to topical antimicrobials (I). Epidemiology in Singapore. Contact Dermatitis. 1989;21:46-8.

6. Fisher NM, Marsh E, Lazova R. Scar-localized secondary to silver sulfadiazine cream. J Am Acad Dermatol. 2003;49:730-2.

7. Vincenzi C, Morelli R, Bardazzi F, Guerra L. Contact dermatitis from chloranphenicol in a leg ulcer cream. Contact Dermatitis.1991;25:64-5.

8. Mowad CM. Allergic contact dermatitis caused by parabens: 2 cases reports and a review. Am J Contact Dermatitis. 2000;11:53-6.
9. Verhaeghe I, Goossens-Dooms A. Multiple sources of allergic contact dermatitis from parabens. Contact Dermatitis. 1997;36:269-70.

10. Pasche-Koo F, Piletta PA, Hunziker N, Hauser C. High sensitization rate to emulsifiers in patients with chronic leg ulcers. Contact Dermatitis. 1994;31:226-8.

11. Wakelin SH, Smith H, White IR, Rycroft RJ, McFadden JP. A restropective analysis of contact dermatitis to lanolin. Br J Dermatol. 2001;145:28-31.

ENDEREÇO PARA CORRESPONDÊNCIA / MAILING ADDRESS:

Rosana Lazzarini

R. Dr. Franco da Rocha, 163 / 102 Perdizes

05015040 São Paulo - SP

Tel./fax: (11) 3875-3196 (11) 3868-4947

E-mail:lazzarini@fototerapia.com.br

Como citar este artigo/How to cite this article: Lazzarini R, Duarte I, Braga JCT, Ligabue SL. Dermatite alérgica de contato a medicamentos de uso tópico: uma análise descritiva. An Bras Dermatol. 2009;84(1):30-4. 